\title{
Development and validation of a nutritional risk screening scale for stroke patients
}

Xu Jihann ${ }^{1}$, Cao Juan ${ }^{1}$, Su Yongjing ${ }^{1}$, Xie Xiaolan ${ }^{1}$, Jin Yuanyuan², Feng Jiezhen ${ }^{1}$, Huang Feiwen ${ }^{1}$, Huang Yongqing ${ }^{1}$, Zhang Xiaoyan ${ }^{3}$

\author{
${ }^{1}$ Department of Neurology, The First Affiliated Hospital, Sun Yat-sen University; \\ Guangdong Provincial Key Laboratory of Diagnosis and Treatment of Major \\ Neurological Diseases, National Key Clinical Department and Key Discipline \\ of Neurology, Guangzhou, China \\ ${ }^{2}$ Neurology Medicine Center, The Seventh Affiliated Hospital (Shen Zhen), Sun Yat-sen \\ University, Guangming (New) Dist., Shenzhen, China \\ ${ }^{3}$ Nursing Department, The First Affiliated Hospital, Sun Yat-sen University, Guangzhou, \\ China
}

Submitted: 2 June 2021; Accepted: 20 July 2021

Online publication: 27 July 2021

Arch Med Sci

DOI: https://doi.org/10.5114/aoms/140352

Copyright $\odot 2021$ Termedia \& Banach

\section{Abstract}

Introduction: Stroke is a global health problem, and around $62 \%$ of stroke patients suffer from malnutrition due to dysphagia, eating speed, or the stress response. This study aimed to develop a Nutritional Risk Screening Scale for Stroke Patients (NRSS-SP).

Material and methods: In the current study we constructed a theoretical framework by combining stroke characteristics, the risk factors of malnutrition in stroke patients, and clinical experience. Then, using the Delphi method, we formed a pool for entries and combined the opinions and suggestions discussed by experts in a research team. Next, we collected all of the data and information, categorized, merged, and split the pool of entry items' contents. Finally, we formed a pre-test scale comprising 11 items after scoring their importance. A clinical investigation of 100 patients was conducted to test the validation of the NRSS-SP.

Results: The pre-test NRSS-SP comprised 10 items in three fields: physical, psychological, and independence. A score was assigned to each factor. Disease severity, the serum level of albumin, and dysphagia were assigned 3 points each, and age $\geq 70$ years was assigned 1 point. The other indicators were assigned 1-3 points according to the evaluation results. The cumulative effect of four factors (depression, anxiety, serum level of albumin, and body mass index (BMI)) was $65.512 \%$. The item-level content validity index (CVI) of the NRSS-SP ranged from 0.081 to 1.000 , and the scale-level CVI was 0.912 . The coefficient of Cronbach's $\alpha$ ranged from 0.822 to 0.911 .

Conclusions: An NRSS-SP (which comprised the National Institutes of Health Stroke Scale score, BMI, serum level of albumin, recent weight loss, recent food intake, dysphagia, age, depression, anxiety, and Barthel index) score $\geq 6.5$ was classified as a malnourishment risk; an NRSS-SP score $<6.5$ denoted normal nutrition.

Key words: stroke, nutrition assessment, malnutrition.

\section{Introduction}

Stroke is a global health problem. Up to $62 \%$ of stroke patients suffer from malnutrition due to dysphagia, eating speed, or the stress response

\author{
Corresponding author: \\ Zhang Xiaoyan \\ Nursing Department \\ The First Affiliated Hospital \\ Sun Yat-sen University \\ 58 Zhongshan Road \\ Guangzhou 510080, China \\ Phone: +86 2087332071 \\ E-mail: zhxyan@mail.sysu. \\ edu.cn, \\ drZhangXiaoyan@hotmail. \\ com
}


[1-3]. According to studies undertaken outside China, $\sim 24 \%$ of stroke patients are malnourished [4]. Malnutrition has been identified as an independent risk factor for stroke and is closely related to adverse clinical outcomes in stroke patients, including increased mortality and morbidity [4]. Despite the fact that malnutrition is under-recognized and under-treated in stroke patients [1], its incidence on admission is believed to be approximately $20 \%$ [2]. The prevalence of malnutrition following an acute stroke, on the other hand, varies significantly, ranging from $6.1 \%$ to $62 \%$ [3]. This large range has been ascribed to a variety of factors, including assessment time, patient characteristics, and, most critically, nutritional evaluation techniques. Malnutrition before and after an acute stroke is linked to longer hospital stays, worse functional outcomes, and higher death rates 3-6 months later $[4,5]$. When compared to ischaemic strokes and intracerebral haemorrhage, subarachnoid haemorrhage $(\mathrm{SAH})$ requires the greatest caloric intake $(\mathrm{ICH})$. As a result, identifying malnutrition quickly after an acute incident using the body mass index (BMI), anthropometric measurements, or laboratory data is critical to minimise bad outcomes [6-8].

The screening of nutritional risk for stroke patients worldwide is based mainly on the universal nutritional status assessment scale, which is not targeted and has different sensitivity $[3,5]$. We aimed to combine the characteristics of stroke and risk factors for malnutrition in stroke patients based on the method for assessment in the Mini-Nutritional Assessment-Short-Form (MNA-SF) [6] and Nutritional Risk Screening 2002 (NRS 2002). We developed a Nutritional Risk Screening Scale For Stroke Patients (NRSS-SP) to evaluate the nutritional status of stroke patients. We provided a basis for a nutritional treatment plan and nursing plan for stroke patients.

\section{Material and methods}

\section{Development of Nutritional Risk Screening Scale for Stroke Patients}

\section{Theoretical framework of the Nutritional} Risk Screening Scale for Stroke Patients

The core of the "whole person" theory expounded by Rogers proposes that humans are a unified and open dynamic whole with the surrounding environment. This theory emphasizes clinical nursing from the standpoint of interaction between people and the environment [7]. The nutritional status of stroke patients is due to various factors. Malnutrition affects the clinical outcomes and recovery of stroke patients. Therefore, guided by this theory, we designed a preliminary draft of an NRSS-SP based on the literature and research on assessment scales of nutritional sta- tus in China and overseas. We referred to the clinical characteristics of stroke and the risk factors of malnutrition after stroke. The scale comprised three fields: physiological, psychological, and in dependence. Hence, an individualized and targeted assessment of the nutritional status of stroke patients was enabled.

\section{Purpose and concept of the Nutritional Risk Screening Scale for Stroke Patients}

The NRSS-SP was created as a specific scale for assessment of the nutritional risk of stroke patients. We undertook a preliminary evaluation of the efficacy of nutritional support in stroke patients to ascertain their nutritional status. According to the factors affecting the nutritional status of stroke patients, medications and dietitians could provide targeted and individualized nutritional treatment programmes and care plans for stroke patients.

The newly developed NRSS-SP should be (i) suitable for qualified staff to use; (ii) concise and brief, with strong practicability and operability, as well as good reliability and validity.

The requirements for writing an entry were that the: (i) item description is concise; (ii) each item clearly reflects a question; (iii) each question reflects the purpose of the design of the NRSS-SP; (iv) items must be representative, have strong independence, and each item can be investigated for each patient.

\section{Establishment of a research team to screen} the pool of entries

The research team comprised various professionals specializing in stroke: physicians, nursing staff, nutritionists, psychological counsellors, and rehabilitation experts. The research team used the definition of malnutrition and referred to the design methods of MNA-SF and NRS 2002 to create the NRSS-SP.

\section{Development of a primary selection scale using the Delphi method}

Experts were invited to review a pool of entries. Experts had to have worked for $\geq 10$ years in neurology, nursing, nutrition, or psychology. Twenty experts from six provinces or cities in China (Guangdong, Beijing, Chongqing, Shanghai, Jilin and Jiangxi) were included. Among them, 80\% (16/20) of experts were from healthcare institutions, and $20 \%(4 / 20)$ of experts were from universities. Stroke specialists comprised 20\% (4/20), neurological-care specialists comprised $20 \%$ (4/20), nutrition experts comprised $50 \%$ (10/20), and psychologists comprised $10 \%(2 / 20)$ of the research team. The mean age of the 20 experts was $48.23 \pm 5.82$ years. Also, $55 \%(11 / 20)$ of experts had a master's degree 
or higher. Experts with 11-19 years of experience constituted $35 \%(7 / 20)$, experts with $20-28$ years of experience constituted $25 \%$ (5/20), and experts with 29-38 years of experience constituted $40 \%$ $(8 / 20)$ of the research team.

Two rounds of expert consultations were conducted using the Delphi method. Anonymity was used to issue questionnaires and emails in two ways to solicit opinions and suggestions from experts on the relevance of each item to the target. After the first round of expert consultation, the data were collated, aggregated, and analysed, combined with expert opinions and item-screening criteria. The research team discussed the indicators to make a second round of expert consultation. Analyses of the reliability of the expert consultation using the Delphi method were carried out using three indicators: positive coefficient, authority level, and coordination degree. Twenty questionnaires were sent for each round of consultation, and all were completed. The expert's judgment was based on 0.82, and the expert's familiarity was 0.88 .

Two criteria were used to delete entries: (i) the arithmetic mean of the importance of the index $<3.5$; (ii) the index had a coefficient of variation $>0.25$. The test for the Kendall rank correlation coefficient was statistically significant. Based on the two rounds of expert consultation and the research team's opinions and suggestions, the entries for the primary selection scale were screened and modified. One item was changed: the serum level of albumin was changed from $<30 \mathrm{~g} / \mathrm{l}$ to $<35 \mathrm{~g} / \mathrm{l}$. One item was added: an increase in the anxiety index. Five items were deleted: (i) the family/friends/love/ marriage relationship is not harmonious; (ii) cannot obtain spiritual encouragement from relatives and friends/economic support/physical assistance; (iii) no healthcare support (public medical support, commercial insurance, or social insurance); (iv) household income is at a low income level; (v) fever. Finally, 11 entries were included.

\section{Formation of a primary Nutritional Risk Screening Scale for Stroke Patients}

We combined the data analysis results of the two rounds of expert consultation and the opinions and suggestions of the research team so that 11 indicators were included in the primary selection scale of the NRSS-SP. The research team selected the corresponding measurement tools for these 11 indicators. Then, they formed a pre-test evaluation form for the NRSS-SP.

\section{Validation study}

\section{Study design}

According to the order of hospital admission, inpatients diagnosed as having a stroke in a neu- rology department in one of the top three hospitals in Guangzhou from July 2017 to February 2018 were investigated.

The inclusion criteria for the study cohort were: (i) stroke was diagnosed in accordance with the diagnostic criteria adopted by the Fourth National Conference on Cerebrovascular Diseases in 1995[8]; (ii) stroke was confirmed by computed tomography or magnetic resonance imaging; (iii) patients were aged $\geq 18$ years or $\leq 80$ years; (iv) patients provided written informed consent to participate in the study.

The exclusion criteria were: (i) death within 3 days of hospital admission; (ii) severe liver or kidney dysfunction; (iii) end-stage chronic disease; (iv) severe mental illness.

The criteria for excluding data were: (i) patients who died during the study; (ii) patients who withdrew voluntarily at any stage of the survey.

For stable and reliable results and accurate estimation of parameters, it has been suggested that the actual sample content should be 5-10-times that of the observed variable [9]. In the present study, the variable in the primary selection scale was 11 , and the sample size was 10 times that of the study variable. The calculated sample size was 110 cases. Considering a sample loss of $10 \%$, the final sample size was 120 cases.

Each department hired a specialist nurse for training. The time of investigation was when the patient was admitted to the hospital. The investigator visited each patient individually, described briefly the purpose of the investigation, obtained consent, and completed the NRSS-SP.

\section{Study group}

Of 120 cases, there were 75 (62.5\%) males and 45 (37.5\%) females. The male:female ratio was $5: 3$. The youngest study participant was aged 28 years, and the oldest was aged 80 years. The mean age of the study cohort was $60.54 \pm 11.47$ years. A total of $70.8 \%$ of stroke patients came to see a physician in a time range of 2 days to 31 days. Also, $84.2 \%$ of patients had a stroke for the first time. The type of stroke was cerebral infarction in $85.8 \%$ of cases. The most common complication of stroke patients was hypertension (23.3\%). Eight (6.7\%) patients paid for treatment at their own expense and 39 (32.5\%) paid using rural cooperative medical care. Figure 1 provides the flowchart of the current study cohort.

\section{Ethical approval}

The current study was approved by the ethical committee of the First Affiliated Hospital, Sun Yat-sen University; Guangdong. Informed consent was obtained from all the participants. 


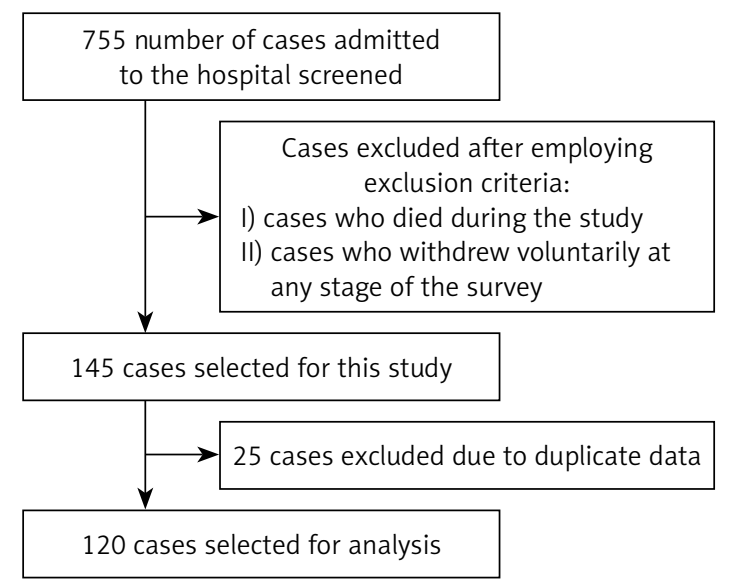

Figure 1. Flowchart for the study cohort

\section{Statistical analysis}

Validity tests

"Structural validity" is a method of validity analysis that reflects the degree of integration of research tools with the theoretical or conceptual framework on which they are based. Factor analysis is used to test the structural validity of a particular scale. Factor analysis uses a few factors to describe multiples, or the relationship between multiple factors, reflecting most of the information in the original data with fewer factors [10]. Factor analysis can transform multiple observed variables into a few unrelated comprehensive indicators to reflect the characteristics and nature of a particular feature [11]. The Kaiser-Meyer-Olkin (KMO) statistic of the NRSS-SP was 0.647. Bartlett's test had $p<0.001$. Hence, a common factor was present between the correlation matrices of the population, which was suitable for factor analysis. Principal component analysis retained a factor with an eigenvalue $>1$ as a common factor, to perform a maximum variance rotation on the initial factor load matrix $[9,12]$. The structural validity of the NRSS-SP was verified by factor analysis. The test principle was: 1) extraction factor characteristic value $>1$; 2) factor cumulative variance contribution rate $>50 \%$; 3 ) item load value $<0.4$ was deleted. The independent $t$-test was used for comparison of variables between baseline and malnutrition stroke patients at 6 months after treatment. $P<0.05$ was considered statistically significant. SPSS V25 was used for statistical analysis.

\section{Content validity}

We employed different methods to ask experts to score the importance of NRSS-SP. Then, we calculated the content validity index of each item and the content validity index of the NRSS-SP.

\section{Reliability test}

The internal consistency of the NRSS-SP and each item was tested using Cronbach's $\alpha$ coefficient.

\section{Calculating malnourishment risk}

A score was assigned to each factor. Disease severity, the serum level of albumin, and dysphagia were assigned 3 points each, and age $\geq 70$ years was assigned 1 point. The other indicators were assigned 1-3 points according to the evaluation results. A score more than 5 was considered to predict a malnourishment risk.

\section{Results}

\section{Structural validity}

\section{First-factor analysis}

Four common factors had an eigenvalue $>1$. The cumulative variance of these four common factors accounted for $60.768 \%$ of the total variance. Therefore, it was considered reasonable to extract four common factors (Table I). We deleted the variable with a load value $<0.4$ (i.e., we delet-

Table I. Factor analysis for the NRSS-SP: the total number of variances explained

\begin{tabular}{|lccc|}
\hline Factor & Eigenvalue & Variance interpretation (\%) & Cumulative contribution (\%) \\
\hline 1 & 2.963 & 26.938 & 26.938 \\
\hline 2 & 1.453 & 13.209 & 40.147 \\
\hline 3 & 1.181 & 10.739 & 50.886 \\
\hline 4 & 1.087 & 9.882 & 60.768 \\
\hline 5 & 0.934 & 8.493 & 69.261 \\
\hline 6 & 0.898 & 8.167 & 77.428 \\
\hline 7 & 0.786 & 7.148 & 84.577 \\
\hline 8 & 0.562 & 5.113 & 89.690 \\
\hline 9 & 0.472 & 4.292 & 93.983 \\
\hline 10 & 0.454 & 4.123 & 98.106 \\
\hline 11 & 0.208 & 1.894 & 100.00 \\
\hline
\end{tabular}


Table II. Factor analysis for the NRSS-SP: rotating factor matrix-1

\begin{tabular}{|lcccc|}
\hline Item & \multicolumn{3}{c|}{ Factor } \\
\cline { 2 - 5 } & $\mathbf{1}$ & $\mathbf{2}$ & $\mathbf{3}$ & $\mathbf{4}$ \\
\hline Dysphagia & 0.789 & -0.014 & 0.014 & -0.243 \\
\hline NIHSS & 0.732 & 0.050 & 0.128 & 0.150 \\
\hline BI index & 0.676 & 0.278 & -0.039 & 0.057 \\
\hline Anxiety score & 0.564 & 0.539 & 0.019 & 0.217 \\
\hline Depression core & 0.414 & 0.701 & -0.037 & 0.225 \\
\hline Serum level of albumin & 0.168 & 0.642 & 0.092 & 0.050 \\
\hline BMI & -0.127 & 0.630 & 0.067 & -0.145 \\
\hline Weight loss & -0.087 & 0.164 & 0.850 & -0.201 \\
\hline Reduced food intake in 1 week & 0.185 & -0.016 & 0.810 & 0.242 \\
\hline Fever & 0.251 & -0.130 & -0.100 & -0.687 \\
\hline Age & 0.279 & -0.066 & -0.069 & 0.656 \\
\hline
\end{tabular}

ed the variable "fever") (Table II). The clinical data showed that among 120 cases, 80 underwent IV thrombolysis, 30 underwent mechanical thrombectomy and 15 underwent both IV thrombolysis and mechanical thrombectomy. Moreover, 86 patients were suffering from anxiety and depression, and 16 had mild symptoms of depression based on the psychologist report.

\section{Second-factor analysis}

After the removal of the fever item, the principal component analysis was undertaken again. The KMO value was 0.659, and Bartlett's test had $p<0.001$, indicating that there was a correlation between the variables and that factor analysis could be undertaken. Factor analysis showed four common factors with an eigenvalue $>1$. The cumulative variance of these four common factors accounted for $65.512 \%$ of the total variance. Therefore, it was rational to extract four common factors (Table III). The load value of 10 items was $>0.4$ on four common factors, so the items after the second-factor analysis were unchanged (Table IV). After the entry's deletion, 10 entries were retained, the KMO statistic was 0.659, and Bartlett's test had $p<0.001$, indicating a correlation between the variables. Hence, indicating our newly developed scale covered the content of the construct to be measured (Table V).

\section{Content validity}

The content validity of the total NRSS-SP was 0.912. The content validity of each item of the NRSS-SP was 0.081-1.000.

\section{Reliability test}

The Cronbach's $\alpha$ coefficient of the total NRSSSP was 0.876 . The Cronbach's $\alpha$ coefficient of each item of the NRSS-SP was 0.822-0.911.

\section{NRSS-SP}

After the clinical test, the pre-experimental table of the NRSS-SP comprised 10 items, including

Table III. NRSS-SP factor analysis - total variance explained

\begin{tabular}{|lccc|}
\hline Factor & Eigenvalues & Variance interpretation rate (\%) & Cumulative contribution rate (\%) \\
\hline 1 & 2.963 & 29.626 & 29.626 \\
\hline 2 & 1.434 & 14.34 & 43.966 \\
\hline 3 & 1.129 & 11.293 & 55.259 \\
\hline 4 & 1.025 & 10.253 & 65.512 \\
\hline 5 & 0.911 & 9.114 & 74.626 \\
\hline 6 & 0.787 & 7.867 & 82.493 \\
\hline 7 & 0.590 & 5.903 & 88.396 \\
\hline 8 & 0.494 & 4.937 & 93.334 \\
\hline 9 & 0.456 & 4.563 & 97.896 \\
\hline 10 & 0.210 & 2.104 & 100 \\
\hline
\end{tabular}


Table IV. NRSS-SP factor analysis - rotating factor matrix-1

\begin{tabular}{|lcccc|}
\hline Item & \multicolumn{3}{c|}{ Factor } \\
\cline { 2 - 5 } & $\mathbf{1}$ & $\mathbf{2}$ & $\mathbf{3}$ & $\mathbf{4}$ \\
\hline Dysphagia & 0.809 & -0.017 & -0.001 & -0.084 \\
\hline NIHSS & 0.783 & 0.052 & 0.113 & 0.093 \\
\hline BI & 0.669 & 0.293 & -0.041 & 0.115 \\
\hline Depression core & 0.353 & 0.739 & -0.022 & 0.232 \\
\hline Serum albumin & 0.139 & 0.654 & 0.096 & 0.027 \\
\hline BMI & -0.142 & 0.615 & 0.065 & -0.151 \\
\hline Anxiety score & 0.522 & 0.572 & 0.029 & 0.231 \\
\hline Reduced food intake in a week & 0.109 & 0.027 & 0.836 & 0.298 \\
\hline Weight loss & -0.036 & 0.135 & 0.832 & -0.299 \\
\hline Age & 0.057 & 0.046 & 0.004 & 0.914 \\
\hline
\end{tabular}

Table V. Contents of each item represented by a common factor

\begin{tabular}{|lr|}
\hline Common factor & Representative aspect \\
\hline 1 & NIHSS, dysphagia, BI \\
\hline 2 & $\begin{array}{r}\text { Post-stroke depression, anxiety, } \\
\text { serum level of albumin, BMI }\end{array}$ \\
\hline 3 & $\begin{array}{r}\text { Weight loss, reduced food intake } \\
\text { within 1 week }\end{array}$ \\
\hline 4 & Age \\
\hline
\end{tabular}

the physiological field, psychological field, and independence field. Disease severity, the serum level of albumin, and dysphagia were assigned 3 points each, and age $\geq 70$ years was assigned 1 point. The other indicators were assigned 1-3 points according to the evaluation results. A total score $\geq 6.5$ suggested a malnourishment risk. A total score $<6.5$ was considered to denote normal nutrition.

\section{Discussion}

\section{The NRSS-SP is rational and scientific}

There are several mechanisms for the deterioration of nutritional status after stroke [13]. Malnutrition is related mainly to dysphagia, neurological impairment, recent weight loss, reduced dietary intake, fever, post-stroke depression, ability to carry out activities of daily living, economic status of the family, and old age $[14,15]$. Malnutrition can lead to changes in body composition and body cell mass. These actions can result in reduced nerve function and affect the clinical outcome of malnutrition. Until now, a universal assessment scale for nutritional status for stroke patients has been lacking.

Based on the theory of the development of scales [9, 10, 12], guides for nutrition scores [6], specialized nutritional assessment scales (e.g., MNA-SF, NRS 2002) [14, 16] and the literature, we began to create the NRSS-SP. After repeated discussions and revisions, primary selections were drawn up. Then, 20 experts from six provinces and cities across China were selected to form a research team, which comprised experts from the fields of neurology, nursing, nutrition, psychology and other fields. After two rounds of expert consultation, the selected items were screened, tested for reliability and validity, combined with the opinions and suggestions of experts, and data were summarized and analysed. Finally, 10 items were constructed to create a questionnaire for nutritional risk in stroke patients. After two rounds of consultation, all 20 experts completed the questionnaire, indicating that the experts were highly motivated and cooperated with our study.

\section{The NRSS-SP has good reliability and validity}

Inspection of the NRSS-SP was carried out in strict accordance with the principles of reliability and validity. Cronbach's $\alpha$ coefficient was employed to reflect the internal consistency of the NRSS-SP. In general, Cronbach's $\alpha$ coefficient is $>0.7$ [12]. The two rounds of expert consultation demonstrated that the NRSS-SP had good content validity.

For verification of structural validity, Table $\mathrm{V}$ shows that the first common factor consisted of three items: National Institutes of Health Stroke Scale (NIHSS) score, dysphagia, and BI index. These items represented the physiological field and independence field. Researchers have shown that the functional defects caused by stroke (e.g., limb spasm, hemifacial spasm, paraesthesia) can lead to instability of the body position and dysphagia, which affects nutrient intake $[17,18]$. The second common factor consisted of four items, depression, anxiety, serum level of albumin, and body mass index (BMI), which represented the psychological field and physiological field. Stroke 
causes impairment of physical function and a decline in the quality of daily life, resulting in a reduction in patients' confidence. Anorexia, incompatibility with treatment, and an increased incidence of malnutrition have been observed after stroke [19-21]. The third common factor comprised two items, weight loss and reduced food intake within 1 week, which represented the physiological field. Stroke patients can have reduced dietary intake and weight loss $[15,22]$. The fourth common factor comprised one item of age, and represented the physiological field. Age $\geq 70$ years was associated significantly with clinical outcomes. Studies have shown that the older the patient, the more pronounced is the aging of the main organs of the body, the worse is the functional reserve, the worse is the gastrointestinal function, and the higher is the incidence of malnutrition [23]. After deleting the fever item, 10 entries were retained with a KMO statistic of 0.659 and Bartlett's test had $p<0.001$, thereby indicating a correlation between the variables. Hence, our newly developed NRSS-SP covered the constructs to be measured.

The entries in our study were the physical field, psychological field, and independence field, and these three fields were related to each other. The results showed that the entries in these four common factors had an intrinsic logical relationship. Therefore, the NRSS-SP that we developed had structural validity.

\section{The NRSS-SP has strong practicability and operability}

Screening for nutritional risk involves determining whether an individual is at risk of malnutrition or determining whether a detailed nutritional assessment is needed [24]. Nutritional risk screening is rapid and identifies people at potential nu- tritional risk. The nutritional status assessment includes anthropometric measurements, dietary surveys, laboratory tests, and comprehensive nutritional assessment [25]. The latter includes the use of various assessment scales for screening of nutritional risk.

Studies on nutritional risk for stroke patients are lacking [26]. Universal scales for various diseases have been developed. The European Society of Parenteral Enteral Nutrition recommends using NRS 2002 and the Malnutrition Universal Screening Tool (MUST) [27] to screen patients at nutritional risk, and MNA-SF is recommended for older people [6]. The German Clinical Nutrition Society recommends NRS 2002, MUST, MNA-SF, and Subjective Global Assessment (SGA) as nutritional screening tools for patients who have suffered an acute stroke. In 1993, SGA began to be used for the nutritional evaluation of patients with liver diseases in China [28]. Studies undertaken in China suggest that NRS 2002, MNA-SF, and PGSGA could be used as screening tools for stroke patients' nutritional risk [29].

There are several limitations to the current study, which need to addressed in future studies. The study included a limited number of cases, and thus it is necessary to conduct a study involving a large population from various regions. The study lacks application of various approaches, including rehabilitation and other therapeutic approaches, to evaluate participants and compare with the predictive scoring value of the current study. The scale developed in the current study comprises 11 items, which should be further elaborated and expanded in future studies (Figure 2).

In conclusion, an NRSS-SP (which comprised the NIHSS score, BMI, serum level of albumin, recent weight loss, recent food intake, dysphagia, age, depression, anxiety, and $\mathrm{BI}$ index) score $\geq 6.5$

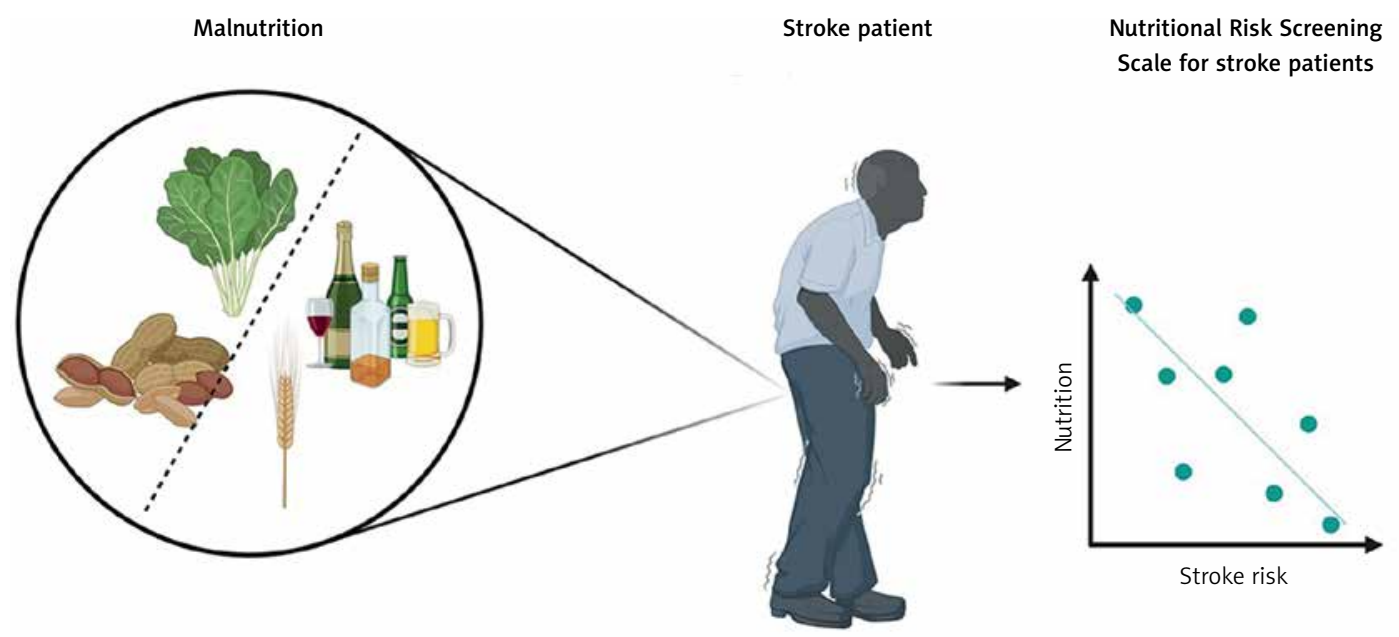

Figure 2. Illustration showing that malnutrition can worsen the condition of a stroke patient. In this study we evaluated the effect of malnutrition on stroke and developed a Nutritional Risk Screening Scale for Stroke Patients (NRSS-SP) 
Table VI. Comparison of variables between baseline at the time when the patient was diagnosed with malnutrition and malnutrition stroke patients at 6 months after treatment

\begin{tabular}{|c|c|c|c|}
\hline Variables & $\begin{array}{c}\text { Baseline } \\
(n=120) \text { mean (SD) }\end{array}$ & $\begin{array}{l}\text { Malnutrition stroke patients } \\
6 \text { months after treatment } \\
(n=120) \text { mean (SD) }\end{array}$ & $P$-value ${ }^{a}$ \\
\hline Body mass index $\left[\mathrm{kg} / \mathrm{m}^{2}\right]$ & $23.1(4.3)$ & $25.1(3.3)$ & $<0.001$ \\
\hline Riboflavin [mg] & $1.6(0.6)$ & $1.8(0.6)$ & 0.243 \\
\hline Albumin $[\mathrm{g} / \mathrm{dl}]$ & $3.1(1.6)$ & $3.6(1.7)$ & $<0.001$ \\
\hline Iron [mg] & $12.1(4.4)$ & $14.4(5.1)$ & $<0.001$ \\
\hline Sodium [mg] & $1952.0(614.4)$ & 2321(412) & $<0.001$ \\
\hline Potassium [mg] & $1176.5(393.7)$ & $1934(234)$ & 0.036 \\
\hline Protein [g] & $51.6(12.4)$ & $67(14.1)$ & $<0.001$ \\
\hline Carbohydrate [g] & $156.9(49.1)$ & $167(41)$ & $<0.001$ \\
\hline
\end{tabular}

andependent $t$-test with significance level $p<0.05$.

was classified as a malnourishment risk; an NRSSSP score $<6.5$ denoted normal nutrition. To reduce the disability and recurrence rate of stroke, the NRSS-SP can: (i) promote communication and cooperation between physicians, nurses, and patients/families; (ii) help to identify the existing and potential nutritional risks of stroke patients; (iii) improve the nutritional level of patients. Figure 2 illustrates and summarizes the concept of the current study (Table VI).

\section{Acknowledgments}

This study was supported by grants from the Southern China International Cooperation Base for Early Intervention and Functional Rehabilitation of Neurological Diseases (2015B050501003), Guangdong Provincial Engineering Center For Major Neurological Disease Treatment, Guangdong Provincial Translational Medicine Innovation Platform for Diagnosis and Treatment of Major Neurological Disease, Guangdong Provincial Clinical Research Center for Neurological Diseases, Guangdong Medical Science and Technology Research Fund Project (Project Number: A2020468), Project Name: Clinical study of the effect of mealstyle enteral nutrition infusion on the nutritional status and complications of stroke patients.

\section{Conflict of interest}

The authors declare no conflict of interest.

\section{References}

1. Ying Z. Study on nutritional status assessment and nutrition intervention in stroke patients with dysphagia. Doctoral thesis. Shan Dong University 2016.

2. Calvo I, Pizzorni N, Gilardone G, et al. Predictors of oral feeding resumption after stroke in a rehabilitation hospital: a retrospective study. J Stroke Cerebrovasc Dis 2019; 28: 1958-70.

3. Nishioka S, Okamoto T, Takayama M, et al. Malnutrition risk predicts recovery of full oral intake among old- er adult stroke patients undergoing enteral nutrition: Secondary analysis of a multicentre survey (the APPLE study). Clin Nutr 2017; 36: 1089-96.

4. Gomes F, Emery PW, Weekes CE. Erratum to: Risk of malnutrition is an independent predictor of mortality, length of hospital stay, and hospitalization costs in stroke patients: Journal of Stroke and Cerebrovascular Diseases 2016:25(4):799-806. J Stroke Cerebrovasc Dis 2016; 25: 2091.

5. Ying Z, Jiang Z. Nutrition screening, nutrition assessment and nutrition intervention were three key steps of nutrition care: the 2011 Clinical Guideline of the American Society for Parenteral and Enteral Nutrition (ASPEN). Chin J Clin Nutr 2012; 20: 261-8.

6. Cederholm T, Bosaeus I, Barazzoni R, et al. Diagnostic criteria for malnutrition - An ESPEN Consensus Statement. Clin Nutr 2015; 34: 335-40.

7. Zheng J, Chen X. Rogers' science of unitary human beings theory and its application in nursing care. Chin Nurs Res 2017; 3: 1-4.

8. Chinese Society of Neurology. Diagnostic criteria of cerebrovascular diseases in China (version 2019). Chin J Neurol 2019; 52: 710-5.

9. Devellis RF. Scale development: theory and applications. Third edition. Chongqing University Press, Chongqing 2016; 75-167.

10. Li Z, Yu L. Nursing Research. People's Medical Publishing House, Beijing 2012; 71-87.

11. Wu S, Pan F. Methods of statistical programming for social sciences. Tsinghua University Press, Beijing 2014; 334-44.

12. Wang C, Luo J. Advanced medical statistics. Science Press, Beijing 2014; 425-34.

13. Wang J, Luo B, Xie Y, et al. Evaluation methods on the nutritional status of stroke patients. Eur Rev Med Pharmacol Sci 2014; 18: 3902-7.

14. Li D, Yongjun W, Shaoshi W, et al. Chinese experts consensus on dysphagia and nutrition management of stroke patients. Chin J Stroke 2013; 8: 973-83.

15. Deng X, Huang L, Hongyan J, et al. Risk factors analysis of undernutrition, complications and poor prognosis in acute stroke patients. J Clin Neurol 2013; 26: 31-4.

16. Professional Committee of cancer nutrition and support therapy of China Anti Cancer Association. Chinese specialist consensus on nutritional management of stroke patients. Chin J Cerebrovasc Dis 2007; 4: 211-3.

17. Wu J. The recovery mechanism of lower limb's motor dysfunction and dysphagia in brain injury patients. Doctoral thesis, Fudan University 2013. 
18. Tang G, Ma R, Qiu D, et al. The effect of enteral nutrition on immune function, nutritional index and neurological deficits in patients with dysphagia after stroke. J Brain and Nervous Dis 2017; 25: 340-3.

19. He Y, Hu Q, Fan L, et al. Research on multi factors analysis and nursing method in the old patients with poststroke depression. J Int Psychiatry 2015; 42: 19-22.

20. Liang J. Investigation of anxiety and depression in acute phase and convalescence patients with stroke and research for its influencing factors. Doctoral thesis, Tianjin Medical University 2015.

21. Yu C, Chen Y, Lv Y, et al. Major depressive disorder in relation with coronary heart disease and stroke in Chinese adults aged 30-79 years. J Peking Univ 2016; 48: 465-71.

22. Corrigan ML, Escuro AA, Celestin J, et al. Nutrition in the stroke patient. Nutr Clin Pract 2011; 26: 242-52.

23. Christner S, Ritt M, Volkert D, et al. Evaluation of the nutritional status of older hospitalised geriatric patients: a comparative analysis of a Mini Nutritional Assessment (MNA) version and the Nutritional Risk Screening (NRS 2002). J Hum Nutr Diet 2016; 29: 704-13.

24. Chinese Society for Oncological Nutrition Supportive Care, The Committee of Rehabilitation and Palliative Care, et al. Nutrition Risk Screening. Electronic J Metabol Nutrition Cancer 2016; 3: 100-1.

25. Chen X, Wen T, Yan L. Research progress of nutritional status and nutritional intervention in patients with tumor radiotherapy. Tianjin J Nurs 2016; 24: 366-8.

26. Royal College of Physicians. National clinical guideline for stroke. Royal College of Physicians, London 2012; 99-249.

27. Wirth R, Smoliner C, Jager M, et al. Guideline clinical nutrition in patients with stroke. Exp Transl Stroke Med 2013; 5: 14.

28. Chen R, Guo J, Feng Y, et al. The application and comparison of method of clinical nutritional assessment SGA. ACTA Nutr Sin 1993; 15: 454-7.

29. He F, Chen C. Comparison of three different subjective assessment tools for nutritional assessment and screening at middle and aged patients with stroke. Parent Enteral Nutr 2014; 21: 358-60. 